\title{
Integrated silicon nitride time-bin entanglement circuits
}

\author{
Xiang Zhang ${ }^{1,2, *}$, Bryn A. Bell ${ }^{1,2}$, Andri Mahendra ${ }^{2,3}$, Chunle Xiong $^{1,2}$, Philip H. W. Leong ${ }^{2,3}$, \\ AND BENJAMIN J. EGGLETON ${ }^{1,2}$ \\ ${ }^{1}$ Centre for Ultrahigh bandwidth Device for Optics Systems (CUDOS), Institute of Photonic and Optical Science (IPOS), School of Physics, University of Sydney, \\ NSW 2006, Australia. \\ ${ }^{2}$ The University of Sydney Nano Institute, University of Sydney, NSW 2006, Australia. \\ ${ }^{3}$ School of Electrical and Information Engineering, University of Sydney, NSW 2006, Australia. \\ *Corresponding author: b.zhang@physics.usyd.edu.au
}

Compiled June 17, 2018

Time-bin entangled photons allow robust entanglement distribution over quantum networks. Integrated photonic circuits positioned at the nodes of a quantum network can perform the important functions of generating highly entangled photons and precisely manipulating their quantum state. In this work, we demonstrate time-bin entangled photon generation, noise suppression, wavelength division and entanglement analysis on a single photonic chip utilizing low-loss doublestripe silicon nitride waveguide structures. Quantum state tomography results show $91 \pm 0.7 \%$ fidelity compared with the ideal state, indicating that highly entangled photons are generated and analyzed. This work represents a crucial step toward practical quantum networks.

(C) 2018 Optical Society of America

OCIS codes: (270.5565) Quantum communications; (250.5300) Photonic integrated circuits.

http://dx.doi.org/10.1364/ao.XX.XXXXXX

\section{INTRODUCTION}

Entanglement plays an essential role in quantum technologies such as quantum communication, quantum computing, and quantum metrology [1]. In particular for quantum communication, entanglement between remote users is a resource that enables quantum key distribution [2,3], quantum secure direct communication [4-7], quantum teleportation [8], dense coding $[9,10]$ and quantum repeaters [11]. Time-bin entangled photons transmitted through optical fiber are a promising approach for distributing entanglement over long distances. In such an approach, quantum bits (qubits) are encoded onto a photon which can exist in either an early or a late arrival time [2]. These quantum states do not decohere during transmission because photons are naturally well-isolated from their environment, and time-bin encoding is highly robust compared to using the polarization or paths of photons $[12,13]$, in terms of stress and temperature fluctuations in fiber. The generation and manipulation of time-bin entanglement usually requires the use of unbalanced interferometers, with a time-delay in one path. Here the use of integrated photonic chips offers intrinsic phase-stability as well as repeatable fabrication of precisely chosen time delays, which are problematic in a fiber or free-space unbalanced interferometer [14]. Meanwhile, the integrated solution imposes stringent requirements on the integration platform. For instance, although the silicon-on-insulator platform has excellent nonlinearity, its propagation loss $(3 \mathrm{~dB} / \mathrm{cm})$ prohibits the construction of long time-delays $[15,16]$. There have been numerous experiments demonstrating the generation of time-bin entanglement using integrated nonlinear elements to create correlated photon pairs, with the unbalanced interferometers either implemented in fiber or on a separate, linear chip [15, 17-19]. However, an integrated photonic chip that includes photon source and linear circuits will open the possibility of developing a robust, scalable and cost-efficient quantum network. To the best of our knowledge, this is the first work that combines nonlinear photon-pair generation with linear processing of time-bin entanglement states on a single chip.

In this work, we utilize low-loss double-stripe silicon nitride waveguide structures to fabricate an integrated photonic chip that includes entangled pair generation, noise suppression, wavelength division and entanglement analysis. After carrying out quantum state tomography (QST), a fidelity of $91 \pm 0.7 \%$ compared with the ideal state indicates highly entangled photons are generated and analyzed in this integrated circuit, demonstrating its potential for practical applications in quantum networks.

\section{EXPERIMENTAL SETUP}

Fig. 1(a) shows the experimental setup used in this work. A $50 \mathrm{MHz}$ mode-locked laser (MLL) generates $10 \mathrm{ps}$ pump pulses (green) centered at $1552.5 \mathrm{~nm}$. Amplified spontaneous emission (ASE) noise from an internal erbium-doped fiber amplifier (EDFA) is filtered by a band pass filter (BPF) with $0.3 \mathrm{~nm}$ full width at half maximum (FWHM). Pump power and polarization are adjusted by an attenuator (ATT) and a polarization controller (PC) respectively, prior to input to the silicon nitride chip.

The chip utilizes low-loss double-stripe silicon nitride waveguides that only support the transverse electric (TE) mode with 

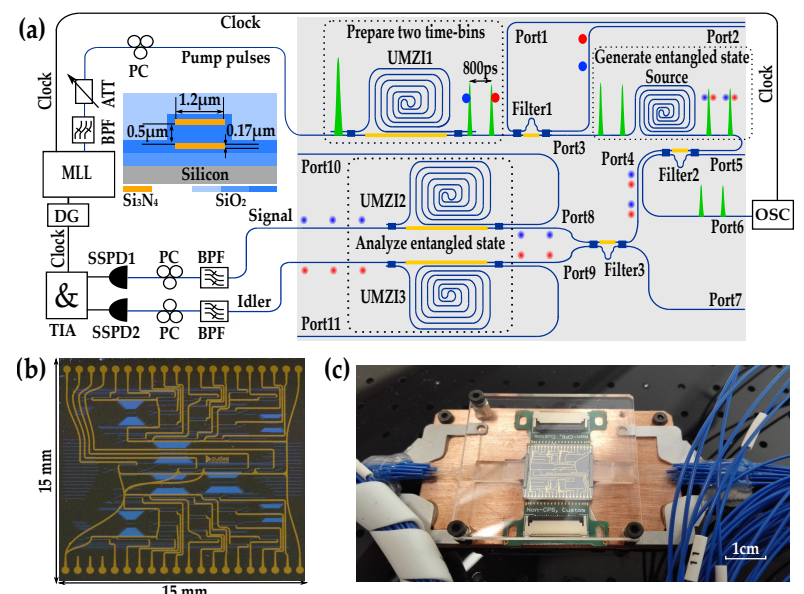

Fig. 1. (a) Experimental setup. MLL: mode-locked laser; BPF: band pass filter; ATT: attenuator; PC: polarization controller; UMZI: unbalanced Mach-Zehnder interferometer; DG: delay generator; OSC: oscilloscope; SSPD: super-conducting single photon detector; TIA: time interval analyzer. Black and blue lines are electronic and optical circuits, respectively. Solid circles are noise photons; Blurred circles are entangled photons; Gold rectangles are thermal phase shifters. (b) Photo of the bare photonic chip. Yellow lines are electronic connections and blue lines are the underlying silicon nitride waveguides. (c) The chip after packaging with printed circuit boards (PCBs) and arrays of polarization maintaining fibers.

$0.2 \mathrm{~dB} / \mathrm{cm}$ propagation loss $[15,16] .<0.1 \mathrm{~dB} / \mathrm{cm}$ propagation loss should be achievable with an optimized fabrication process [20]. Its cross-sectional structure and dimensions are shown as an inset in Fig. 1(a). The schematic of the photonic chip is also illustrated in Fig. 1(a). First, an unbalanced Mach-Zehnder interferometer (UMZI1), with 800 ps time delay between short and long paths, splits each pump pulse into two identical pulses which define two time-bins (early and late). The additional loss $(3 \mathrm{~dB})$ introduced by the long arm $(14 \mathrm{~cm})$ of UMZI1 is compensated by adjusting the coupling ratio of the input and output coupler so that there is equal power transmitted through each arm [15], and similar for UMZI2 and 3. A thermal phase-shifter (gold) in UMZI1 is used to tune the relative phase between early and late pump pulses, $\theta_{p}$. In the long arm of UMZI1, the strong pump pulses will create noise photons (blue and red circles) by spontaneous four-wave mixing (SFWM) and spontaneous Raman scattering (SpRS). A Mach-Zehnder interferometer with a small length imbalance of $176 \mu \mathrm{m}$ between paths is used to create a filter (Filter1) which transmits the pump pulses but rejects noise at the wavelengths which will be used for the entangled photons. After tuning the phase shifter, the spectrum of Filter1 is shown as the magenta line in Fig. 2 (a) and it indicates the spectrum of the signal (blue) and idler (red) where the entangled photons will be created (centered at $1548.5 \mathrm{~nm}$ and $1556.5 \mathrm{~nm}$ ). Comparing with the input spectrum of amplified spontaneous emission (ASE) noise (black), the transmission loss at pump wavelength is around $7 \mathrm{~dB}$. Considering the coupling loss to fibre of $3 \mathrm{~dB}$ per facet, this suggests Filter1 has a $1 \mathrm{~dB}$ insertion loss. Then a $6.5 \mathrm{~cm}$ spiral waveguide is used to generate correlated signal and idler photon pairs via SFWM, a $\chi^{(3)}$ nonlinear process with quadratic dependence on the peak power of the pump pulses. A characterization of photon pair generation in this source is given in Ref.[16]. The photon pairs are created in
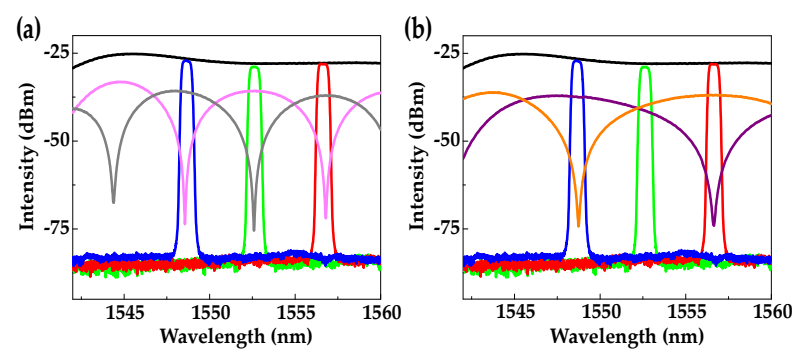

Fig. 2. Spectra of Filter1-3. Black, blue, green and red lines represent the ASE, signal, pump and idler spectra respectively. (a) Spectra of Filter1 (magenta) and Filter2 (gray). (b) Spectrum of Filter3; purple and orange lines represent the signal and idler outputs, respectively. All filters exhibit $>30 \mathrm{~dB}$ extinction ratios.

a coherent superposition state between each time-bin, so their state can be expressed as $\left(|E\rangle_{s}|E\rangle_{i}+e^{2 i \theta_{p}}|L\rangle_{s}|L\rangle_{i}\right) / \sqrt{2}$, where $|E\rangle_{x}$ and $|L\rangle_{x}$ represent a photon in the early or late time-bin, respectively, and $x=s, i$ indicates signal and idler wavelengths.

After photon-pair generation, Filter2 with $176 \mu \mathrm{m}$ length difference is used to separate the pump pulses from the entangled photon pairs. Filter2's spectrum is shown as the gray line in Fig. 2(a) and its insertion loss at signal and idler are 1 and $2 \mathrm{~dB}$ respectively. Pump pulses are sent to an output port (Port6) and monitored by an oscilloscope (OSC). The pump power inside the spiral circuits is estimated by temporarily replacing OSC with a power meter. The measured power of $0.8 \mathrm{~mW}$ indicates the average pump power and the peak power inside the spiral are $2.1 \mathrm{~mW}$ and $4.2 \mathrm{~W}$, respectively, due to the coupling loss ( $3 \mathrm{~dB})$ and the propagation loss $(1.3 \mathrm{~dB})$. Filter 3 with $88 \mu \mathrm{m}$ length difference is used to divide the entangled signal and idler photons, with the spectra of the two output ports shown in Fig. 2(b). The insertion loss of Filter 3 is around $2 \mathrm{~dB}$. Each filter is particularly designed to perform noise suppression or wavelength demultiplexing based on the characterization of the spiral waveguide in Ref.[16]. The filters have a tuning range of around $800 \mathrm{GHz}$ for their central frequency, using thermal phase shifters. In the measurement, signal and idler are then sent to two separate UMZIs (UMZI2 and 3) with time-delays which match UMZI1. These are used to measure and analyze the time-bin qubits. A photon originally in one of two time-bins can arrive at one of three times after passing through an UMZI. A photon previously in the $|E\rangle$ $(|L\rangle)$ state can never be detected at the latest (earliest) of the three arrival times. Hence detecting a photon at the earliest or latest time makes a projective measurement onto the $|E\rangle$ or $|L\rangle$ state. However a photon detected in the middle arrival time could have originated from either time-bin, and so makes a projective measurement onto a superposition state: $\left(|E\rangle_{x}+e^{i \theta_{x}}|L\rangle_{x}\right) / \sqrt{2}$, where $\theta_{x}$ is the relative phase between the arms of the UMZI. Each relative phase is controlled by a thermal phase-shifter in the corresponding UMZI. The ability to project time-bin qubits onto coherent superposition states makes it possible to verify that they are entangled and to carry out QST.

The photons are coupled off-chip and residual noise from the pump laser is removed by high isolation $(\geq 60 \mathrm{~dB})$ and low loss (2 dB) BPFs with $0.3 \mathrm{~nm}$ FWHM. The polarizations are adjusted by PCs to maximize the detection efficiency of super-conducting single photon detectors (SSPDs) to around 50\%. The dark counts and the timing-jitter of the SSPDs are around $200 \mathrm{~Hz}$ and $50 \mathrm{ps,}$ respectively. A time interval analyzer (TIA, ID800) with 81 ps time-resolution is connected to three inputs: signal and idler 


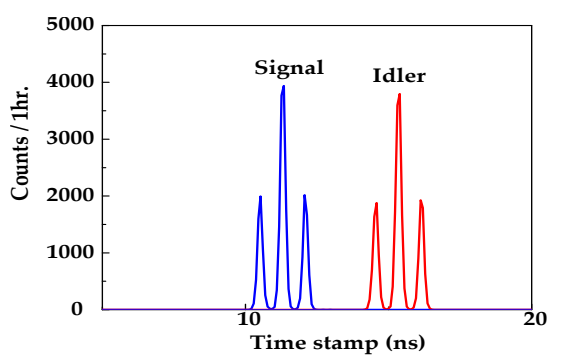

Fig. 3. Histogram of signal and idler arrival times after postprocessing time-bin information, relative to the laser clock.

SSPDs, and the laser clock, which is used for synchronization. The repetition rate of the clock signal is down-converted by a delay generator (DG) to $1 \mathrm{MHz}$ since the original $50 \mathrm{MHz}$ clock would saturate the TIA. The photonic chip before and after bonding are shown in Fig. 1 (b) and (c), respectively.

\section{METHODS AND RESULTS}

The difference between signal and idler detection times is used to identify photons which originated from the same laser pulse and so constitute an entangled pair; the down-converted clock is sufficient to identify which of the three possible arrival times a given photon was detected in, relative to the original laser clock. An example histogram is given in Fig. 3, showing the relative arrival times. Three peaks are visible for both signal and idler detections. The width of the peaks is due to the combined timing jitter of the SSPDs, the TIA, and the down-converted clock. It can be seen that this jitter is small enough compared to the $800 \mathrm{ps}$ time separation to allow each of the three peaks to be clearly distinguished.

First, two photon interference is used to calibrate the pump phase $\theta_{p}$, which is varied whilst keeping $\theta_{s}$ and $\theta_{i}$ fixed. Fig. 4 shows the interference fringe in the two photon counts, postselected on both signal and idler arriving in their central histogram peak. A visibility of $88 \%$ is obtained from a sinusoidal fit (the dashed line). At a maxima in this count rate, $2 \theta_{p}=\theta_{s}+\theta_{i}$. Hence we fix the pump phase at the first maxima, where $V^{2}=64$, and carry out QST by varying $\theta_{S}$ and $\theta_{i}$, following the method of Ref.[21]. The selected phase of $\theta_{s}$ and $\theta_{i}$ are $\pi / 2$ and $-\pi / 2$, respectively, in addition to zero for both phases.

The coincidence counts at each specific phase setting have been measured for one hour to suppress statistical uncertainty. After post-processing, each coincidence count is classified into one of the 16 state projections required for QST [21]. In the time-bin basis, the total coincidence counts where signal and idler arrive in the same time-bin (early or late) are 10.2 and $11.5 \mathrm{k}$, compared to $<100$ for the two combinations where they arrive at different times, demonstrating a high level of correlation. These remaining uncorrelated counts are thought to be noise contributed by detector dark counts, Raman noise and multiphoton noise. The coincidence counts in the two superpositionmeasurement-bases expected to show constructive two photon interference are around 10-10.5k. In the other bases, the counts are evenly distributed at around 5-6k. The average coincidence rate is $7 \mathrm{~Hz}$, which could be improved four-fold by placing detectors at the unused outputs of the signal and idler UMZIs. Each channel has a collection efficiency of around $-16 \mathrm{~dB}$, including 3-4 $\mathrm{dB}$ from filters 2 and 3, $3 \mathrm{~dB}$ from the UMZI, 3-4 dB coupling loss to fiber, $2 \mathrm{~dB}$ loss from off chip filters, $1 \mathrm{~dB}$ loss from the polarization controller, and $3 \mathrm{~dB}$ loss from detector inefficiency. This

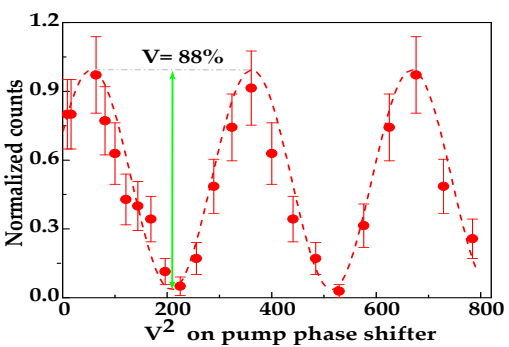

Fig. 4. Two-photon quantum interference with $88 \%$ visibility (V); Red dots represent the results after post-selecting and count normalization; the dashed line is a sinusoidal fit. Error bars show one standard deviation, assuming Poisson count statistics.

suggests an entangled pair generation rate of $45 \mathrm{kHz}$, around $10^{-3}$ pairs per pump pulse. Considering its potential applications in quantum network, further improvements are required to significantly boost the coincidence rate. This could include the use of a ring resonator to enhance the pair generation rate for a given pump power. Other possibilities include integrating low-loss and high isolation (>95 dB) filters [22], increasing the pump repetition rate [23], integrating high efficiency detectors (or number-resolving detectors) [24]. The mode-matching to fiber could also be considerably improved using inverse tapers of the output waveguides or polymer mode-converters.[25]

The density matrix is linearly constructed by the coincidence counts based on the results of the aforementioned state classification, then a maximum likelihood estimation approach is adopted to find a physical density matrix which best fits the experimental results $[21,26]$. The resulting density matrix has $91 \pm 0.7 \%$ fidelity compared with the ideal state, as shown in Fig. 5 (a) and (b). This is consistent with the two photon interference visibility of $88 \%$ measured in Fig. 4 according to Eq.(3) in Ref.[27]. The fidelity is well above the classical threshold of $50 \%$, as well as the $70.7 \%$ threshold to violate a Bell's inequality, confirming highly entangled photon pairs are generated and analyzed in this integrated silicon nitride circuit.

\section{DISCUSSION AND CONCLUSIONS}

The small deviation between the measured state and the ideal state is likely due to the influence of noise sources, such as SpRS noise in the silicon nitride, and errors in the beam-splitter ratios and phase-settings on the chip, partially due to the thermal crosstalk between each heater. These problems could be significantly mitigated by cryogenic conditions and a dynamic feedback system. Compared with silicon nanowires, nonlinear photon pair generation in the double-stripe silicon nitride waveguides requires further improvement. Dispersion engineering and using a ring resonator instead of spiral waveguides could significantly enhance the generation efficiency and the signal-to-noise-ratio (SNR), such as at least 40-fold enhancement of the photon generation efficiency and CAR $>270$ with negligible Raman noise [16]. Another strategy to enhance the photon generation rate is to increase the pump repetition rate, where the upper limit is define by the timing-jitter of the detection system, because the minimum delay to distinguish two time-bins is determined by the timing-jitter [23].

Apart from stability and scalability, reproducibility is another crucial advantage of CMOS compatible platforms. Another silicon nitride circuit on the same chip with an identical layout has 
(a)

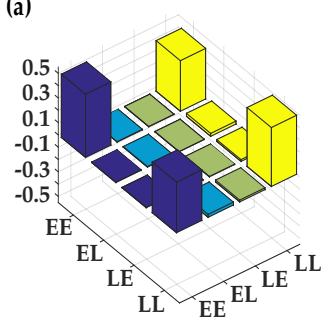

(c)

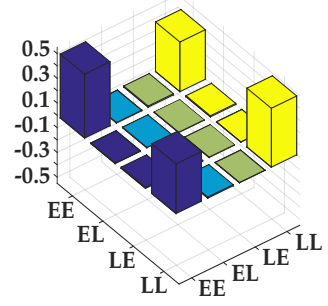

(b)

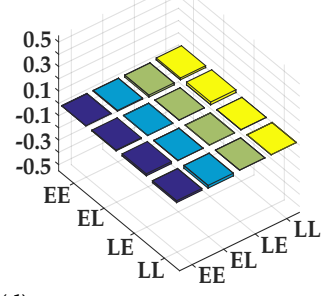

(d)

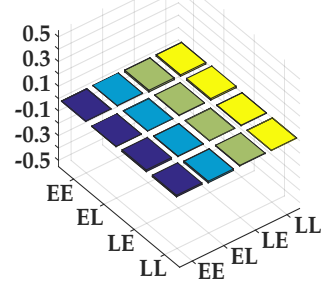

Fig. 5. Density matrix of time-bin entangled photon pairs generated in two samples after maximum likelihood estimation. (a) and (b) show the real and imaginary parts of density matrix in the first sample, respectively.(c) and (d) show the real and imaginary parts of density matrix in the second sample, respectively. EE, EL, LE and LL represent $|E\rangle_{s}|E\rangle_{i},|E\rangle_{s}|L\rangle_{i}$, $|L\rangle_{s}|E\rangle_{i}$ and $|L\rangle_{s}|L\rangle_{i}$, respectively.

been characterized in this work. After implementing maximum likely-hood estimation, $89 \pm 0.6 \%$ fidelity is obtained for the second sample and its density matrix is shown in Fig. 5 (c) and (d). The $2 \%$ fidelity discrepancy between two sources originates from slightly different coupling ratios in UMZIs. The fidelity results of these two chips further confirm the reproducibility of this architecture. In the future, the external filters and SSPDs could also be integrated as high extinction filters and near-unity efficiency SSPDs have recently been demonstrated in a silicon nitirde platform $[22,28]$. This could allow scaling to more complicated functions, e.g. distributed quantum machine learning and multi-photon entangled state generation [29, 30]. Here, integrated silicon nitride circuits not only offer a compact and robust platform to generate entangled photons locally, but also allow entangled photons' transmission and analysis between separate photonic chips, such as using Port 10 and 11 shown in Fig. 1 to analyze entangled photons from other nodes in the quantum network.

In this work, time-bin entangled photon pairs are generated and analyzed for the first time on a single integrated chip containing silicon nitride photonic circuits. The entangled states are analyzed by QST and the maximum likely-hood estimation indicates highly entangled photons are generated with $91 \pm 0.7 \%$ fidelity compared with the ideal state. These results pave the way for a cost-effective and reliable entanglement source for building practical quantum networks.

\section{FUNDING INFORMATION}

Centre of Excellence (CUDOS CE110001018); Laureate Fellowship (FL120100029); Discovery Early Career Researcher Award (DE1201100226); Indonesia Endowment Fund for Education (LPDP); This work is partially supported by Huawei Technologies Inc.

We would like to thank LioniX for fabricating the silicon nitride chip. We acknowledge funding support from the Aus-

tralian Research Council (ARC) and Huawei Technologies Inc.

\section{REFERENCES}

1. R. Horodecki, P. Horodecki, M. Horodecki, and K. Horodecki, Reviews of Modern Physics 81, 865 (2009).

2. I. Marcikic, H. de Riedmatten, W. Tittel, V. Scarani, H. Zbinden, and N. Gisin, Physical Review A 66, 062308 (2002).

3. A. K. Ekert, Physical Review Letters 67, 661 (1991).

4. G. L. Long and X. S. Liu, Physical Review A 65, 032302 (2002).

5. F.-G. Deng, G. L. Long, and X.-S. Liu, Physical Review A 68, 042317 (2003).

6. W. Zhang, D.-S. Ding, Y.-B. Sheng, L. Zhou, B.-S. Shi, and G.-C. Guo, Physical Review Letters 118, 220501 (2017).

7. F. Zhu, W. Zhang, Y. Sheng, and Y. Huang, Science Bulletin 62, 1519 (2017).

8. C. H. Bennett, G. Brassard, R. Jozsa, A. Peres, and W. K. Wootters, Physical Review Letters 70, 1895 (1993).

9. C. H. Bennett and S. J. Wiesner, Physical Review Letters 69, 2881 (1992).

10. K. Mattle, H. Weinfurter, P. G. Kwiat, and A. Zeilinger, Physical Review Letters 76, 4656 (1996).

11. H.-J. Briegel, W. Dür, J. I. Cirac, and P. Zoller, Physical Review Letters 81, 5932 (1998).

12. N. Matsuda, H. Le Jeannic, H. Fukuda, T. Tsuchizawa, W. J. Munro, K. Shimizu, K. Yamada, Y. Tokura, and H. Takesue, Scientific Reports 2, 817 (2012).

13. J. W. Silverstone, R. Santagati, D. Bonneau, M. J. Strain, M. Sorel, J. L. O'Brien, and M. G. Thompson, Nature Communications 6, 7948 (2015).

14. I. Marcikic, H. de Riedmatten, W. Tittel, H. Zbinden, M. Legré, and N. Gisin, Physical Review Letters 93, 180502 (2004).

15. C. Xiong, X. Zhang, A. Mahendra, J. He, D.-Y. Choi, C. J. Chae, D. Marpaung, A. Leinse, R. G. Heideman, M. Hoekman, C. G. H. Roeloffzen, R. M. Oldenbeuving, P. W. L. van Dijk, C. Taddei, P. H. W. Leong, and B. J. Eggleton, Optica 2, 724 (2015).

16. X. Zhang, Y. Zhang, C. Xiong, and B. J. Eggleton, Journal of Optics 18, 074016 (2016).

17. C. Reimer, M. Kues, P. Roztocki, B. Wetzel, F. Grazioso, B. E. Little, S. T. Chu, T. Johnston, Y. Bromberg, L. Caspani, D. J. Moss, and R. Morandotti, Science 351, 1176 (2016).

18. Y. H. Li, Z. Y. Zhou, Z. H. Xu, L. X. Xu, B. S. Shi, and G. C. Guo, Physical Review A 94, 1 (2016).

19. Y.-H. Li, Z.-Y. Zhou, L.-T. Feng, W.-T. Fang, S.-I. Liu, S.-K. Liu, K. Wang, X.-F. Ren, D.-S. Ding, L.-X. Xu, and B.-S. Shi, Physical Review Applied 7, 064005 (2017).

20. K. Wörhoff, R. G. Heideman, A. Leinse, and M. Hoekman, Advanced Optical Technologies 4, 189 (2015).

21. H. Takesue and Y. Noguchi, Optics Express 17, 10976 (2009).

22. A. W. Elshaari, I. E. Zadeh, A. Fognini, M. E. Reimer, D. Dalacu, P. J. Poole, V. Zwiller, and K. D. Jöns, Nature Communications 8, 379 (2017).

23. X. Zhang, B. Bell, M. Pelusi, J. He, W. Geng, Y. Kong, P. Zhang, C. Xiong, and B. J. Eggleton, Applied Optics 56, 8420 (2017).

24. M. D. Eisaman, J. Fan, A. Migdall, and S. V. Polyakov, Review of Scientific Instruments 82, 071101 (2011).

25. P.-I. Dietrich, M. Blaicher, I. Reuter, M. Billah, T. Hoose, A. Hofmann, C. Caer, R. Dangel, B. Offrein, U. Troppenz, M. Moehrle, W. Freude, and C. Koos, Nature Photonics 12, 241 (2018).

26. D. F. V. James, P. G. Kwiat, W. J. Munro, and A. G. White, Physical Review A 64, 052312 (2001).

27. H. de Riedmatten, I. Marcikic, J. A. W. van Houwelingen, W. Tittel, H. Zbinden, and N. Gisin, Physical Review A 71, 050302 (2005).

28. C. Schuck, W. H. P. Pernice, and H. X. Tang, Applied Physics Letters 102, 051101 (2013).

29. Y. B. Sheng and L. Zhou, Science Bulletin 62, 1025 (2017).

30. J.-W. Pan, Z.-B. Chen, C.-Y. Lu, H. Weinfurter, A. Zeilinger, and M. Żukowski, Reviews of Modern Physics 84, 777 (2012). 


\section{FULL REFERENCES}

1. R. Horodecki, P. Horodecki, M. Horodecki, and K. Horodecki, "Quantum entanglement," Reviews of Modern Physics 81, 865-942 (2009).

2. I. Marcikic, H. de Riedmatten, W. Tittel, V. Scarani, H. Zbinden, and N. Gisin, "Time-bin entangled qubits for quantum communication created by femtosecond pulses," Physical Review A 66, 062308 (2002).

3. A. K. Ekert, "Quantum cryptography based on Bell's theorem," Physical Review Letters 67, 661-663 (1991).

4. G. L. Long and X. S. Liu, "Theoretically efficient high-capacity quantumkey-distribution scheme," Physical Review A 65, 032302 (2002).

5. F.-G. Deng, G. L. Long, and X.-S. Liu, "Two-step quantum direct communication protocol using the Einstein-Podolsky-Rosen pair block," Physical Review A 68, 042317 (2003).

6. W. Zhang, D.-S. Ding, Y.-B. Sheng, L. Zhou, B.-S. Shi, and G.-C. Guo, "Quantum Secure Direct Communication with Quantum Memory," Physical Review Letters 118, 220501 (2017).

7. F. Zhu, W. Zhang, Y. Sheng, and Y. Huang, "Experimental long-distance quantum secure direct communication," Science Bulletin 62, 15191524 (2017).

8. C. H. Bennett, G. Brassard, R. Jozsa, A. Peres, and W. K. Wootters, "Teleporting an Unkown Quantum State via Dual Classical and \{EPR\} Channels," Physical Review Letters 70, 1895-1899 (1993).

9. C. H. Bennett and S. J. Wiesner, "Communication via one- and twoparticle operators on Einstein-Podolsky-Rosen states," Physical Review Letters 69, 2881-2884 (1992).

10. K. Mattle, H. Weinfurter, P. G. Kwiat, and A. Zeilinger, "Dense Coding in Experimental Quantum Communication," Physical Review Letters 76, 4656-4659 (1996).

11. H.-J. Briegel, W. Dür, J. I. Cirac, and P. Zoller, "Quantum Repeaters: The Role of Imperfect Local Operations in Quantum Communication," Physical Review Letters 81, 5932-5935 (1998).

12. N. Matsuda, H. Le Jeannic, H. Fukuda, T. Tsuchizawa, W. J. Munro, K. Shimizu, K. Yamada, Y. Tokura, and H. Takesue, "A monolithically integrated polarization entangled photon pair source on a silicon chip," Scientific Reports 2, 817 (2012).

13. J. W. Silverstone, R. Santagati, D. Bonneau, M. J. Strain, M. Sorel, J. L. O'Brien, and M. G. Thompson, "Qubit entanglement between ring-resonator photon-pair sources on a silicon chip," Nature Communications 6, 7948 (2015).

14. I. Marcikic, H. de Riedmatten, W. Tittel, H. Zbinden, M. Legré, and N. Gisin, "Distribution of Time-Bin Entangled Qubits over $50 \mathrm{~km}$ of Optical Fiber," Physical Review Letters 93, 180502 (2004).

15. C. Xiong, X. Zhang, A. Mahendra, J. He, D.-Y. Choi, C. J. Chae, D. Marpaung, A. Leinse, R. G. Heideman, M. Hoekman, C. G. H. Roeloffzen, R. M. Oldenbeuving, P. W. L. van Dijk, C. Taddei, P. H. W. Leong, and B. J. Eggleton, "Compact and reconfigurable silicon nitride time-bin entanglement circuit," Optica 2, 724 (2015).

16. X. Zhang, Y. Zhang, C. Xiong, and B. J. Eggleton, "Correlated photon pair generation in low-loss double-stripe silicon nitride waveguides," Journal of Optics 18, 074016 (2016).

17. C. Reimer, M. Kues, P. Roztocki, B. Wetzel, F. Grazioso, B. E. Little, S. T. Chu, T. Johnston, Y. Bromberg, L. Caspani, D. J. Moss, and R. Morandotti, "Generation of multiphoton entangled quantum states by means of integrated frequency combs," Science 351, 1176-1180 (2016).

18. Y. H. Li, Z. Y. Zhou, Z. H. Xu, L. X. Xu, B. S. Shi, and G. C. Guo, "Multiplexed entangled photon-pair sources for all-fiber quantum networks," Physical Review A 94, 1-7 (2016).

19. Y.-H. Li, Z.-Y. Zhou, L.-T. Feng, W.-T. Fang, S.-I. Liu, S.-K. Liu, K. Wang, X.-F. Ren, D.-S. Ding, L.-X. Xu, and B.-S. Shi, "On-Chip Multiplexed Multiple Entanglement Sources in a Single Silicon Nanowire," Physical Review Applied 7, 064005 (2017).

20. K. Wörhoff, R. G. Heideman, A. Leinse, and M. Hoekman, "TriPleX: a versatile dielectric photonic platform," Advanced Optical Technologies 4, 189-207 (2015).

21. H. Takesue and Y. Noguchi, "Implementation of quantum state tomography for time-bin entangled photon pairs," Optics Express 17, 10976 (2009).
22. A. W. Elshaari, I. E. Zadeh, A. Fognini, M. E. Reimer, D. Dalacu, P. J. Poole, V. Zwiller, and K. D. Jöns, "On-chip single photon filtering and multiplexing in hybrid quantum photonic circuits," Nature Communications 8, 379 (2017).

23. X. Zhang, B. Bell, M. Pelusi, J. He, W. Geng, Y. Kong, P. Zhang, C. Xiong, and B. J. Eggleton, "High repetition rate correlated photon pair generation in integrated silicon nanowires," Applied Optics 56, 8420 (2017)

24. M. D. Eisaman, J. Fan, A. Migdall, and S. V. Polyakov, "Invited Review Article: Single-photon sources and detectors," Review of Scientific Instruments 82, 071101 (2011).

25. P.-I. Dietrich, M. Blaicher, I. Reuter, M. Billah, T. Hoose, A. Hofmann, C. Caer, R. Dangel, B. Offrein, U. Troppenz, M. Moehrle, W. Freude, and C. Koos, "In situ 3D nanoprinting of free-form coupling elements for hybrid photonic integration," Nature Photonics 12, 241-247 (2018).

26. D. F. V. James, P. G. Kwiat, W. J. Munro, and A. G. White, "Measurement of qubits," Physical Review A 64, 052312 (2001).

27. H. de Riedmatten, I. Marcikic, J. A. W. van Houwelingen, W. Tittel, H. Zbinden, and N. Gisin, "Long-distance entanglement swapping with photons from separated sources," Physical Review A 71, 050302 (2005)

28. C. Schuck, W. H. P. Pernice, and H. X. Tang, "NbTiN superconducting nanowire detectors for visible and telecom wavelengths single photon counting on Si3N4 photonic circuits," Applied Physics Letters 102 051101 (2013).

29. Y. B. Sheng and L. Zhou, "Distributed secure quantum machine learning," Science Bulletin 62, 1025-1029 (2017).

30. J.-W. Pan, Z.-B. Chen, C.-Y. Lu, H. Weinfurter, A. Zeilinger, and M. Żukowski, "Multiphoton entanglement and interferometry," Reviews of Modern Physics 84, 777-838 (2012). 